\title{
How accurately do general practitioners and students estimate coronary risk in hypercholesterolaemic patients?
}

Lars Backlund Family Medicine Stockholm, Karolinska Institutet, Stockholm, Sweden Johan Bring Statisticon, Uppsala, Sweden and Lars-Erik Strender Family Medicine Stockholm, Karolinska Institutet, Stockholm, Sweden

\begin{abstract}
Recent guidelines on hyperlipidaemia recommend the calculation of individual coronary risk, at least for patients without previous cardiovascular disease. Although tables and computer programs exist, the estimates are often made on an intuitive basis. The aim of the present work was to study Swedish general practitioners' (GPs) and medical students' ability to estimate the 10-year risk of coronary events for hypercholesterolaemic patients. Two hundred randomly selected Swedish GPs and 73 medical students in their final year of medical school were asked to estimate coronary risk for 10 written case descriptions with different cholesterol levels (at least 5.5 $\mathrm{mmol} / \mathrm{l}$ ) and combinations of other risk factors. Both primary and secondary prevention cases were represented. The risk estimates were compared with the estimates from the Framingham equation and a Swedish equation. The interindividual differences in estimated risk were remarkable for both GPs and students. Both GPs and students underestimated coronary risk, especially for high-risk patients. GPs tended to be more accurate than students in ranking the cases. Cases with previous coronary heart disease were not recommended treatment to the extent that the guidelines recommend. Both GPs and students were quite accurate in estimating absolute risk increase attributable to successive cholesterol increases in a scenario with other risk factors kept constant. It was concluded that GPs and medical students need help to differentiate more accurately between patients at high and low coronary risk, and greater effort should be made to communicate the advantages and difficulties involved in multiple risk assessment. The requirements for decision support are discussed. More evidence is needed on the validity of the Framingham equation for new population samples.
\end{abstract}

Key words: guidelines; hypercholesterolaemia; hyperlipidaemia; primary health care; risk assessment

\section{Introduction}

The doctor's task in the prevention of coronary heart disease (CHD) is to identify and evaluate the individual patient's risk factors and to guide the patient to informed and rational decisions. One controversial problem in the area of preventive cardiology is that of identifying individuals at such

Address for correspondence: Lars Backlund, MD, Family Medicine Stockholm, Karolinska Institutet, Alfred Nobels alle' 12, SE-141 83, Huddinge Sweden. Email: lars.backlund@ klinvet.ki.se elevated risk of future cardiovascular events that treatment for their cholesterol values would be justified. Treatment with statins has been shown to reduce the incidence of CHD, both in primary prevention (Shepherd et al., 1995) and for individuals with already manifest coronary heart disease, i.e., secondary prevention (The $4 \mathrm{~S}$ study, 1994). Statins have generally proved to be cost-effective for all groups of patients in the secondary prevention situation, whereas cost-effectiveness in primary prevention seems to vary based on the patient's sex and age (Jönsson, 2001; Prosser et al., 2000). There is accordingly fairly good agreement 
between different guidelines on treatment in the secondary prevention situation: pharmacological treatment should be initiated even for moderately elevated cholesterol values $(5-5.5 \mathrm{mmol} / \mathrm{l})$. How the primary prevention situation should be managed is less clear, and more room should be left for the individual doctor's clinical decision making. Guidelines, if they are used, can improve both the clinical process and the outcome (Grimshaw and Russell, 1993). GPs seem to have a more positive attitude toward the use of guidelines than doctors from other specialities (Watkins et al., 1999). However, the effect of guidelines on doctors' clinical behaviour has often been questioned (e.g., Lomas et al., 1989). In a controlled trial, dissemination of cholesterol guidelines was shown to have no impact in general practice (van der Weijden et al., 1999) with regard to testing patients at increased cardiovascular risk. Many factors have been suggested as determinants for adherence to guidelines, including attributes of the guidelines themselves (Grol et al., 1998), factors associated with dissemination and implementation (Mansfield, 1995), and even reimbursement (Bjerrum et al., 2001).

In addition to knowledge of the guidelines, decisions on cholesterol treatment should ideally also be based on information about the outcomes of intervention trials and on some familiarity with the concept of individual risk assessment (Simon et al., 1997). Individuals at high risk of developing CHD have more to gain from treatment, and their elevated risk may stem from small contributions from each of several different risk factors (Alderman, 1995; 1996). On the other hand, a moderate increase in blood cholesterol or blood pressure, for example, in the absence of other risk factors may represent a negligible risk.

The recent joint European guidelines on hyperlipidaemia (Wood et al., 1998), based on the Framingham formula (Anderson et al., 1991), explicitly recommend the calculation of individual risk for patients with hypercholesterolaemia in the primary prevention situation and suggest that individuals with a $20 \%$ risk of a coronary event within 10 years should be candidates for pharmacological treatment.

Multiple risk assessments are thus recommended, but it is unclear to what extent such estimates are actually made in clinical practice and, when they are, by what methods. Risk estimates can be derived from tables (Wallis et al., 1997; Wood et al., 1998) or from computer programs, or they can be made subjectively.

Our research question was how Swedish general practitioners (GPs), who do most of the screening for risk factors, and medical students make estimations of the coronary risk for patients with hypercholesterolaemia. If doctors generally overestimate the risk, this could result in drugs being recommended for too many patients, and for underestimation the opposite would be true. We selected case descriptions with a wide range of coronary-risk levels, and both primary and secondary prevention cases were included. Numerical (absolute) risk rather than categorized risk was used, as new guidelines advocate the calculation of absolute risk. Our hypothesis was that GPs would be more accurate, owing to intuitive learning from experience with their own patients and to more exposure to results from relevant clinical trials. Also, we wanted to study how willing GPs and students would be to recommend pharmacological treatment for cases with different risk levels and different patterns of risk factors.

\section{Method}

\section{Participating doctors and students}

GPs:

Two hundred Swedish primary care physicians were randomly selected. All were specialists in family medicine. A postal inquiry was used, with two written reminders.

Students: Seventy-three students in their final year of medical school who were enrolled in the family-medicine course were asked to fill in the questionnaire in connection with a seminar. In contrast to the GPs, their questionnaires were not coded, and thus no reminders were sent, nor were any data about background characteristics collected.

\section{Written case simulations}

The cases consisted of two parts, with a total of 10 written case simulations. An overview of the cases and variables is given in the upper part of Table 1 under 'Results'.

The first part (cases 1-6) contained patients with 
Table 1 Summary of the 10 cases. Risk estimates (Framingham, GPs and students) in percentages. For each case, the GPs' and students' willingnesses to to treat with a drug are shown in percentages and whether pharmacological treatment is recommended by Swedish and joint European guidelines. Risk estimate was not required for case no. 7

\begin{tabular}{|c|c|c|c|c|c|c|c|c|c|c|}
\hline Case no. & 1 & 2 & 3 & 4 & 5 & 6 & 7 & 8 & 9 & 10 \\
\hline Age & 41 & 56 & 66 & 70 & 51 & 61 & 55 & 55 & 55 & 55 \\
\hline $\begin{array}{l}\text { Sex (M male, } \\
\text { F female) }\end{array}$ & $\mathrm{F}$ & $\mathrm{M}$ & $\mathrm{F}$ & $\mathrm{M}$ & $\mathrm{F}$ & M & $\mathrm{M}$ & M & $\mathrm{M}$ & M \\
\hline Hypertension & No & No & No & No & No & No & No & No & No & No \\
\hline Diabetes & No & No & Yes & No & No & No & No & No & No & No \\
\hline Smoker & No & No & No & No & Yes & Yes & No & No & No & No \\
\hline Total cholesterol (mmo/l) & 7.2 & 5.9 & 6.9 & 6.0 & 6.5 & 8.2 & 5.0 & 6.5 & 8.0 & 6.5 \\
\hline LDL (mmo/l) & 5.3 & 4.3 & 4.6 & 4.1 & 4.3 & 6.3 & & & & \\
\hline $\mathrm{HDL}(\mathrm{mmo} / \mathrm{l})$ & 1.2 & 1.1 & 1.1 & 1.0 & 1.0 & 0.9 & & & & \\
\hline Primary/secondary prevention & Prim & $\mathrm{Sec}$ & Prim & $\mathrm{Sec}$ & Prim & Prim & Prim & & & \\
\hline Framingham risk & 4 & & 27 & & 14 & 33 & 12 & 16 & 19 & 16 \\
\hline Estimated median risk GPs & 2 & 15 & 10 & 20 & 5 & 10 & 5 & 7 & 15 & 8 \\
\hline Estimated median risk students & 5 & 10 & 10 & 20 & 8 & 20 & 5 & 14 & 20 & 10 \\
\hline $\begin{array}{l}\text { Percentage of GPs inclined to } \\
\text { treat }\end{array}$ & 16 & 39 & 57 & 49 & 11 & 68 & & 0 & 73 & \\
\hline $\begin{array}{l}\text { Percent of students inclined to } \\
\text { treat }\end{array}$ & 41 & 56 & 54 & 45 & 19 & 86 & & 33 & 93 & \\
\hline $\begin{array}{l}\text { Pharmacological treatment } \\
\text { (Swedish guidelines) }\end{array}$ & No & Yes & No & Yes & No & Consider & No & No & Consider & \\
\hline $\begin{array}{l}\text { Pharmacological treatment (joint } \\
\text { European guidelines) }\end{array}$ & No & Yes & Yes & Yes & No & Yes & No & No & No? & \\
\hline
\end{tabular}

at least $5.5 \mathrm{mmol} / \mathrm{l}$ in total cholesterol. The cases were selected from a sample of patients described elsewhere (Backlund et al., 2000), and represented authentic, primary care patients. The variables presented were compatible both with the variables in the Framingham equation (Anderson et al., 1991) and more recent Swedish material (Berglund etal., 1996), with the following modifications. LDL (low-density lipoprotein) is not included in the two equations but was specified in the case descriptions, as it plays a central role in the Swedish guidelines. To avoid the confusion that could have occurred when judgements on pharmacological treatment were required, only cases with normal blood pressure values were selected, and the diastolic value was set at $80 \mathrm{~mm} \mathrm{Hg}$. Left-ventricular hypertrophy is specified in the Framingham equation but was excluded, as reliable information was difficult to retrieve from the records, and the value was set at zero in the equation (thus possibly underestimating the true risk). Information about a family history of cardiovascular disease is important for risk judgement but was not included in this study on the grounds that this risk factor is not explicitly included in the Framingham formula and that reliable information was often difficult to find in the records.

Cases 1, 3, 5 and 6 represented the primary prevention situation, and cases 2 and 4 concerned patients with previous CHD. For these latter two, the 'true risk' cannot be derived from the Framingham equation, as only subjects free from CHD were included in the Framingham study. The reason for including secondary prevention cases is the high clinical significance as far as cholesterol treatment is concerned.

The second set of cases concerned isolated hypercholesterolaemia. Case 7 was a 'baseline' patient with no clinical risk factors, and no risk estimate was required for this case. Case 8 represented the same case but with a moderate elevation in total cholesterol, and case 9 the same case with a marked increase. Finally, in case 10 the cholesterol value from case 9 was supposed to have been reduced pharmacologically to the levels in case 8 .

The task of the GPs and the students was to estimate the risk of future CHD disease within 10 years. For persons with already existing CHD, the relevant future incident was a new manifestation of the disease. Instead of the risk as a percentage, 
we asked for an estimate of the number of persons, out of a hypothetical group of 100 persons with the same risk factors, that would have CHD within 10 years. There is evidence that information presented as frequencies rather than as probabilities facilitates risk judgements (Gigerenzer, 1996). An example of a case description is shown in Figure 1.

The GPs and students were asked not to use any decision support tools for risk assessment, and the GPs were asked whether any such support was available at the clinic.

The study was approved by the local ethics committee.

\section{Statistical methods}

The 10-year risk was calculated from the Framingham formula (Anderson et al., 1991). The risk estimates from the Swedish material (Berglund et al., 1996) were made by the 'Risk Score 99' computer program, available as a floppy disc from the Hässle Medical Company. Student's t-test was used for differences in age between responders and nonresponders (GPs), the chi-square test for the association between sex and response rate (GPs), the Mann-Whitney test for the difference between medians (GPs and students), and Friedman's test for investigation of a possible difference between the two groups in response patterns across the cases. Correspondence of the individual judgements with the prediction equations was described by Spearman's rank correlation.

\section{Results}

\section{Response rate}

Two hundred GPs were included in the study. Of the nonresponders, 18 had changed work places or were not on duty for other reasons. No infor- mation was available for four of them. Of the remaining 182 possible responders, 84 (46\%) returned the questionnaires. Of these, 15 answered only the questions about pharmacological treatment. There were no significant differences between responders and nonresponders regarding age or sex. Only nine GPs had access to a decision support tool for risk calculation.

Students: Fifty-seven of the 73 students answered the questionnaire $(78 \%)$.

\section{Overall response pattern}

The interindividual differences were remarkable. For example, all the ratings of seven GPs were lower than $10 \%$, whereas the ratings of another eight GPs were all above 10\%. A median rating was calculated for each GP and student for the first seven cases representing independent and separate patients (cases 8-10 were elaborated from case 7). The medians did not differ significantly between GPs and students. Friedman's test for differences in response pattern across the cases did not show any significant differences between GPs and students. Therefore, no separate tests for individual cases were performed.

Three sets of patients are differentiated below: primary prevention cases (1, 3, 5 and 6), secondary prevention cases ( 2 and 4$)$, and cases representing isolated hypercholesterolaemia (7-10).

\section{Primary prevention (cases 1, 3, 5 and 6)}

Figure 2 shows the distribution of ratings as boxplots for each of the primary prevention cases. The Framingham risks are marked. The risks were underestimated by both GPs and students, except for case 1 , representing the lowest risk.

\section{Secondary prevention (cases 2 and 4)}

Figure 3 shows boxplots for the cases with manifest CHD. Corresponding estimates cannot be

Case 1. 41-year-old women with normal blood pressure $(80 \mathrm{~mm} \mathrm{Hg})$ and without diabetes. No coronary heart disease or other atherosclerotic disease. Smoker.

Laboratory values: Total cholesterol $7.2 \mathrm{mmol} / 1$. LDL $5.3 \mathrm{mmol} / \mathrm{l}$. HDL $1.2 \mathrm{mmol} / 1$.

My answer persons would have CHD within 10 years

Would you recommend a lipid-lowering drug? No Yes 


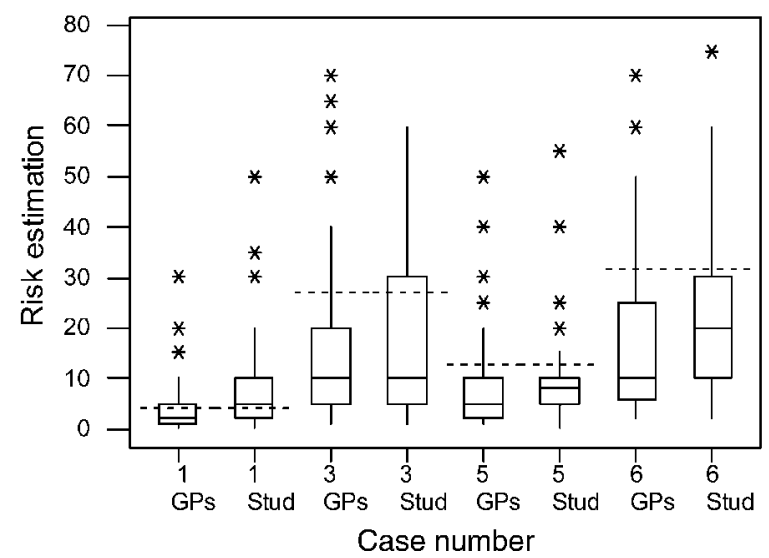

Figure 2 Boxplots of primary prevention cases, ratings by GPs and students. Dashed line denotes the risks estimated from the Framingham equation. The lower and upper limits of the boxes represent the third and first quartiles of the observations and the lines in the boxes are the medians. The 'whiskers' are drawn to the highest and lowest values. When these are extreme, they are marked with asterisks

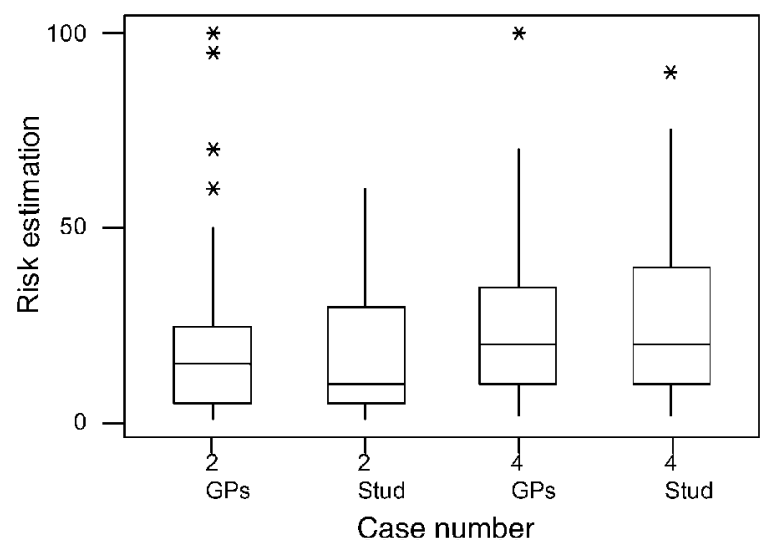

Figure 3 Boxplots of secondary prevention cases. GPs and students. For an explanation of the diagram, see the legend of Figure 2

derived from the Framingham equation, as only individuals free from heart disease were included in the Framingham study. (The Swedish equation includes previous coronary heart disease, but the risks refer to myocardial infarction and not to coronary heart disease in a broader definition.) It has been suggested that the presence of coronary heart disease would increase the risk by at least one category in the risk chart with categories $<5$,
$5-10,10-20,20-40$ and $<40 \%$. The resulting risk would then be roughly around $35 \%$ for case 2 and $45 \%$ for case 4 . This indicates a tendency, both for students and for GPs, to underestimate the risks for the secondary prevention cases at least as much as for the primary prevention cases.

\section{Isolated hypercholesterolaemia (cases 7-10)}

The 10-year coronary risk for the reference case (no. 7), a 55-year-old male with a cholesterol value of $5.0 \mathrm{mmol} / \mathrm{l}$ and no other risk factors, was $12 \%$ according to the Framingham equation, assuming an HDL (high-density lipoprotein) level of 1.1 $\mathrm{mmol} / \mathrm{l}$. An increase to $6.5 \mathrm{mmol} / \mathrm{l}$ (no. 8) elevates the calculated risk to $16 \%$, i.e., an absolute risk difference of $4 \%$. The mean risk difference across GPs was 3\% and across students it was 5\%. A cholesterol increase for the same reference case, from 5.5 to $8.0 \mathrm{mmol} / \mathrm{l}$, gives a Framingham risk increase of $7 \%$ (from 12 to $19 \%$ ). In this scenario, the GPs and students estimated risk increases that were close to the 'true' values $(10 \%$ and $11.5 \%$, respectively). Lowering the cholesterol level from $8.0 \mathrm{mmol} / \mathrm{l}$ to $6.5 \mathrm{mmol} / \mathrm{l}$ with pharmacological treatment was estimated by both GPs and students to result in the same risk as initially having a level of $6.5 \mathrm{mmol} / \mathrm{l}$.

\section{Accuracy}

The rank correlation between actual and predicted risk values is a measure of accuracy. The Framingham equation restricts this comparison to the primary prevention cases, while the Swedish equation includes secondary prevention as well. The difference in outcome measures (CHD vs. myocardial infarction) will probably not affect the ranking of the cases. The two prediction equations give exactly the same ranks to the primary prevention cases. For these cases the median rank correlation was 0.89 for GPs and 0.80 for students, and the difference was not significant. When both primary and secondary prevention cases were included in the Swedish equation, the GPs had a significantly higher median rank correlation $(0.79)$ than the students $(0.68), \mathrm{p}=0.022$.

\section{Willingness to treat}

Table 1 summarizes the different cases, the median ratings by GPs and students, the Framingham estimates and the percentage of respondents willing to prescribe a lipid-lowering drug. 
The case with the highest proportion of respondents willing to treat was number 9 , representing isolated, moderate-to-severe hypercholesterolaemia ( $8.0 \mathrm{mmol} / \mathrm{l})$; almost every student and three GPs out of four were inclined to treat. On the other hand, only about half the respondents were willing to treat the two cases with previous CHD but only moderate elevations of cholesterol (nos. 2 and 4). According to the guidelines, these two patients should be recommended pharmacological treatment. One-third of the students but none of the GPs were inclined to treat a 55-year-old male with mild-to-moderate $(6.5 \mathrm{mmol} / \mathrm{l})$, isolated hypercholesterolaemia (no. 8). More than twice as many students as GPs were inclined to treat the case with the lowest risk (no. 1).

\section{Discussion}

Spontaneous written comments from the GPs indicated lack of time and lack of familiarity with absolute risk estimates as reasons for not responding. The only postal inquiry known to us with absolute estimates of CHD risks (Friedman et al., 1996) resulted in about the same response rate $(40 \%)$. The higher satisfactory response rate for the students in our study can be explained by the different response situations.

Each written case was presented to the participating doctors and students in an identical way but resulted in a remarkable spread in risk estimates. This finding is of clinical relevance if there is a correspondence between the doctor's risk judgement and what is actually communicated to the patient.

Both GPs and students underestimated the risk for high- and moderate-risk patients. The few previous investigations of doctors' judgements of future coronary risk tend to show overestimation, at least when absolute risk estimation is required and the true risks are rather low (Friedman et al., 1996; Grover et al., 1995; Tape and Wigton, 1989). The tendency to underestimate risk has also been shown for a community sample of people, where self-assessed risk and objectively assessed risk of cerebrovascular disease were compared (Niknian et al., 1989). If both patients and doctors underestimate risks, this may lead to lower levels of screening and treatment than desirable. The importance of directing pharmacological treatment to the 'right' patients is underscored by the increasing cost of lipid-lowering drugs. In Sweden, statins showed an increase in daily doses per 1000 inhabitants per day from 11.6 in 1997 to 43.9 in 2001, and in 2002 they were also the largest contributor to the increase in drug costs (statistics from Swedish National Pharmacy Agency, www.apoteket.se, 2003).

Only nine out of 84 GPs answered that they had access to some kind of risk assessment tool in their practice. The reason for this low figure is not evident from the present investigation. At the time of this study, no national distribution of a risk assessment tool had taken place in Sweden. Local implementation and availability of risk assessment tools probably varies substantially among different practices. Other obstacles to the more widespread use of formal risk assessments may also be present. Whereas some decision rules from guidelines can be stored in the doctor's memory, numerical risk assessment requires the chart or computer program to be retrievable at the right moment, and the procedure may be experienced as complicated and time consuming. Moreover, some doctors may feel uncomfortable with concepts like absolute risk and relative risk.

There were small differences between doctors and students, although the GPs seemed to do better in ranking the cases. Perhaps no real competence in estimating absolute risk is acquired through clinical practice, owing to each doctor's relatively small number of patients with coronary events, and to a lack of training in risk assessment. Another explanation may be that the competence acquired in evaluating the risk levels of individual patients is insufficiently captured by the method used in our study.

The students' great willingness to treat patients with isolated hypercholesterolaemia is hardly in accordance with current guidelines. Both students and GPs showed too small an inclination to treat patients with CHD. We have showed this in a previous study with the same type of patient material and a judgement analysis design; about one-fourth of a sample of GPs did not even include CHD in their decision strategy for patients with hypercholesterolaemia (Backlund et al., 2000).

How well the results reflect the accuracy of risk estimation depends on how well the Framingham equation can be generalized to other population samples. It has been shown that the equation yields 
satisfactory predictions for a representative population sample in the USA (Leaverton et al., 1987) and for different northern European populations (Haq et al., 1999). It has been demonstrated that for a Swedish population, the Framingham values seem to give risk estimates that are too high for individuals at low or intermediate risk, while giving risk estimates that are quite close to true estimates for individuals at high risk (SBU, 1995).

A conclusion from the present study is that doctors and students should be helped to differentiate more accurately between patients at high and low coronary risk, especially with respect to the different treatment indications for primary and secondary prevention. Greater effort should be made to communicate to GPs and students the need for, as well as the difficulties in, multiple risk assessment. One practical task is to construct a decision-support instrument that is easily attached to traditional guidelines, that has risk factors compatible with the guidelines, and that is easy to retrieve and use. Computerized guidelines with a decision-support instrument closely linked to the computerized patient record may be a good solution. Another conclusion is that we need more evidence on the usefulness of the Framingham equation for new population samples.

\section{References}

Alderman, M.H. 1995: Quantifying cardiovascular risk in hypertension. Hypertension 13, 519-27.

Alderman, M.H. 1996: Absolute cardiovascular risk: the basis for deciding to treat. American Journal of Nephrology 16, 182-89.

Anderson, K.M., Wilson, P.W.F., Odell, P.M. and Kannel, W.B. 1991: An updated coronary risk profile. A statement for health professionals. Circulation 83, 356-62.

Backlund, L., Danielsson, B., Bring, J. and Strender, L-E. 2000: Factors influencing GPs' decisions on the treatment of hypercholesterolaemic patients. Scandinavian Journal of Primary Health Care 18, 88-93.

Berglund, G., Eriksson, K., Israelsson, B., Kjellström, T., Lindgärde, F., Mattiasson, I., Niisson, J., Å. and Stavenow, L. 1996: Cardiovascular risk groups and mortality in an urban Swedish male population: the Malmö preventive project. Journal of Internal Medicine 239, 489-97.

Bjerrum L., Larsen J. and Kragstrup, J. 2001: Guidelines accompanied by changes in reimbursement. Scandinavian Journal of Primary Health Care 19, 158-62.

Friedman, P.D., Brett, A.S. and Mayo-Smith, M.F. 1996: Differences in generalists' and cardiologists' perceptions of cardiovascular risk and the outcomes of preventive therapy in cardiovascular disease. Annals of Internal Medicine 124, 414-21.
Gigerenzer, G. 1996: The psychology of good judgment: frequency formats and simple algorithms. Medical Decision Making 16, 273-80.

Grimshaw, J.M. and Russell, I.T. 1993: Effect of clinical guidelines on medical practice: a systematic review of rigorous evaluations. Lancet 27, 1317-22.

Grol, R., Dalhuijsen, J., Thomas S., in 't Veld C., Rutten, G. and Mokkink, H. 1998: Attributes of clinical guidelines that influence use of guidelines in general practice: observational study. British Medical Journal 317, 858-61.

Grover, S.A., Lowensteyn, I., Esrey, K.I., Steinert, Y., Joseph, L. and Abrahamowicz, M. 1995: Do doctors accurately assess coronary risk in their patients? Preliminary results of the coronary health assessment study. British Medical Journal 310, 975-78.

Haq, U.I., Ramsay, L.E., Yeo, W.W., Jackson, P.R. and Wallis, E.J. 1999: Is the Framingham risk function valid for northern European populations? A comparison of methods for estimating absolute coronary risk in high risk men. Heart 81, 40-46.

Jönsson, B. 2001: Economics of drug treatment: for which patients is it cost effective to lower cholesterol? Lancet 358, 1251-56.

Leaverton, P.E., Sorlie, P.D., Kleinman, J.C., Dannenberg, A.L., Ingster-Moore, L., Kannel, W.B. and CornoniHuntley, J.C. 1987: Representativeness of the Framingham risk model for coronary heart disease mortality: a comparison with a national cohort study. Journal of Chronic Disease 40, 775-84.

Lomas, J., Anderson, G.M., Domnick-Pierrre, K., Vayda, E., Enkin, M.W. and Hannah, W.J. 1989: Do practice guideleines guide practice? The effects of concensus statement on the practice of physicians. New England Journal of Medicine 321, 1306-11.

Mansfield, C.D. 1995: Attitudes and behaviours towards clinical guidelines: the clinicians' perspective. Quality in Health Care 4, 250-55.

Niknian, M., McKinlay, S.M., Rakovwski, W. and Carleton, R.A. 1989: A comparison of perceived and objective CVD risk in a general population. American Journal of Public Health 79, 1653-54.

Prosser, L.A., Stinnett, A.A., Goldman, P.A., Williams, L.W., Hunik, M.G., Goldman, L. and Weinstein, M.C. 2000: Costeffeftiveness of cholesterol-lowering therapies according to selected patient characteristics. Annals of Internal Medicine 132, 769-79.

No authors listed. Randomized trial of cholesterol lowering in 4444 patients with coronary heart disease: The Scandinavian Simvastatin Survival Study (4S) 1994. Lancet 344, 1383-89.

SBU - The Swedish Council on Technology Assessment in Health Care. Moderately elevated blood pressure 1995. Journal of Internal Medicine 238 (suppl), 737.

Shepherd, J., Cobbe, S., Ford, I., Isles, C., Lorimer, A., MacFarlane, P.W., McKillop, J.H. and Packard, C.J. 1995: Prevention of coronary heart disease with Pravastatin in men with hypercholesterolaemia. New England Journal of Medicine $333,1301-06$.

Primary Health Care Research and Development 2004; 5: 145-152 
Simon, A., Megnien, J. and Levenson, J. 1997: Coronary risk estimation and treatment of hypercholesterolaemia. Circulation 96, 2449-52.

Tape, T.G. and Wigton, R.S. 1989: Medical students' and residents' estimates of cardiac risk. Medical Decision Making 9, 170-75.

Wallis, E., Ramsay, L., Jackson, P., Yeo, W. and Williamson, R. 1997: Drug therapy for coronary heart disease: the Sheffield table. Lancet 350, 1852-54.

Watkins, C., Harvey, I., Langley, C., Gray, S. and Faulkner, A. 1999: General practitioners' use of guidelines in the consul- tation and their attitudes to them. British Journal of General Practice 49, 11-15.

van der Weijden, T., Grol, R. and Knottnerus, J.A. 1999: Feasibility of a national cholesterol guideline in daily practice. A randomized controlled trial in 20 general practices. International Journal for Quality in Health Care 11, 131-37.

Wood, D., DeBacker, G., Faegerman, O., Graham, I., Mancia, G. and Pyörälä, K. 1998: Prevention of coronary heart disease in clinical practice. Summary of recommendations of the second joint task force of European and other societies on coronary heart prevention. European Heart Journal 80, 1-10. 